\title{
Determinación de Factor Reumatoide en Barrio de Juigalpa, Chontales
}

\author{
Yuber Ariel Lazo Guerrero ${ }^{1}$ \\ Deyling Ana Miranda Mairena² \\ Carlos Eduardo López Jirón ${ }^{3}$ \\ Marcos Jesús Aguilar Miranda ${ }^{4}$
}

\section{RESUMEN}

El Factor Reumatoide (FR) es una prueba inmunológica orientativa a una artritis reumatoide. (Goldsby \& Kindt \& Osborne \& Kudy, 2004). Según Restrepo (2007). La determinacion del Factor Reumatoide por el método de aglutinación puede aparecer positivo en enfermedades inmunitarias, entre ellas tenemos: Artritis Reumatoide, Lupus Eritemaso Sistémico, Tuberculosis, Síndrome de Sjogren, Mononucleosis Infecciosa e Infecciones Virales. El estudio es cuantitativo prospectivo de corte transversal, en él participaron 40 mujeres de 40-60 años de edad. El objetivo del estudio es determinar la presencia del factor reumatoide (FR) en mujeres de 40-60 años de edad; debido a que la AR puede presentarse a cualquier edad, pero la mayoría de los casos se encuentran entre la tercera edad, afectando principalmente al sexo Femenino (Restrepo, 2007). Las variables en estudio (edad, ocupación y estado de salud), fueron obtenidas a través del instrumento la encuesta, la cual se les aplicó a 40 mujeres. La determinación del FR se lleva a cabo mediante la realización del método de aglutinación por partículas de látex. Las muestras analizadas se encontraron negativas; no obstante, esto no descarta la probabilidad que desarrollen artritis reumatoide; ya que, desempeñan distintas labores que son factores desencadenantes de la enfermedad, podría obedecer a una combinación de aspectos inmunes, ambientales y genéticos; en donde las articulaciones dañadas son las muñecas, dedos, los codos, hombro, las caderas, rodillas, tobillos, y dedos de los pies.(Restrepo,2007).

Palabras claves: Factor Reumatoide, Aglutinación, Articulaciones, Artritis Reumatoide.

Recibido: 29 de noviembre de 2016

Aceptado: 20 de diciembre de 2016

1 FAREM-Chontales, UNAN-Managua.Correo electrónico: yuberlazo.guerrero@gmail.com

2 FAREM-Chontales, UNAN-Managua. Correo electrónico: deyanamirandamairena@yahoo.es

3 FAREM-Chontales, UNAN-Managua. Correo electrónico: carlosrojas230496@gmail.com

4 FAREM-Chontales, UNAN-Managua. Correo electrónico: aguilarmarcos_10@yahoo.com 


\title{
Determination of Rheumatoid Factor in Juigalpa, Chontales
}

\begin{abstract}
Rheumatoid Factor (RF) is an immunological test for rheumatoid arthritis. (Goldsby \& Kindt \& Osborne \& Kudy, 2004). According to Restrepo (2007), the determination of the Rheumatoid Factor by the method of agglutination can appear positive in immune diseases, among them we have: Rheumatoid Arthritis, Systemic Lupus Eritemaso, Tuberculosis, Sjogren's Syndrome, Infectious Mononucleosis and Viral Infections. The study is a prospective cross-sectional quantitative study involving 40 women aged 40-60 years. The aim of the study is to determine the presence of rheumatoid factor (RF) in women aged 40-60 years; as RA can occur at any age, but most cases are among the elderly, mainly affecting the female sex (Restrepo, 2007). The variables under study (age, occupation and health status) were obtained through surveys, which were applied to 40 women. The determination of FR is carried out by the latex particle agglutination method. The analyzed samples were negative; however, this does not rule out the likelihood of developing rheumatoid arthritis, as they perform different tasks that are triggers of the disease, could be due to a combination of immune, environmental and genetic aspects, where the damaged joints are the wrists, fingers, elbows, shoulder, hips, knees, ankles, and toes (Restrepo, 2007).
\end{abstract}

Keywords: Rheumatoid factor, Agglutination, Joints, Rheumatoid arthritis. 


\section{INTRODUCCIÓN}

El presente artículo está fundamentado en un tema inmunológico en mujeres que habitan en el barrio Nuevo Amanecer de la ciudad de Juigalpa, Chontales en el periodo de Septiembre a Noviembre del año 2016.

El sistema inmunológico es el mecanismo defensa que actúa en un múltiples funciones, que ha venido evolucionando con el fin de proteger al organismo de microorganismos patógenos, cuya función es identificar a las sustancias extrañas y microorganismos que invaden el cuerpo y permitir así su eliminación.

El Factor Reumatoide es un autoanticuerpo dirigido contra la fracción cristalizable de la inmunoglobulina G humana; donde, se detecta a través de una prueba inmunológica. Se encuentra en el suero de $70 \%$ de los pacientes con artritis reumatoide, pero no es exclusivo de esta enfermedad, pues se encuentra en otras enfermedades; Por eso, su positividad no asegura el diagnóstico de artritis reumatoide y necesita correlacionarse con el cuadro clínico.(Castresana, 2004).La detección continúa siendo la principal prueba de laboratorio en la orientación de la artritis reumatoide, puede aparecer antes del inicio de la enfermedad, pero generalmente no se detecta hasta uno o dos años después de éste. Vidal (2010)

\section{FUNDAMENTACIÓN TEÓRICA}

El factor reumatoide consta de anticuerpos de tipo IgM dirigidos contra la región Fc de las $\mathrm{IgG}$, también existen anticuerpos de tipo $\operatorname{IgG}, \operatorname{IgA}$ e incluso $\operatorname{IgE}$.

Los anticuerpos (Acs) son moléculas de naturaleza proteica, cuya función es identificar a las sustancias extrañas y microorganismos que invaden el cuerpo y permitir así su eliminación mediante la acción de otras moléculas también proteicas conocidas como complemento, o bien promoviendo el proceso de fagocitosis. (Gauehff, 2007).

\section{Factor Reumatoide}

Es un autoanticuerpo generalmente del tipo IgM, dirigido contra el fragmento Fc de las inmunoglobulinas IgG. El factor reumatoide se puede presentar en enfermedades reumáticas y no reumáticas, ha sido el autoanticuerpo más asociado a la artritis reumatoide. ( Mendoza \& Biosca, 2015).

Se encuentra en el suero en un 70\% de los pacientes con artritis reumatoide, pero no es exclusivo de esta enfermedad, pues se encuentra en otras enfermedades difusas del tejido conjuntivo como el lupus eritematoso sistémico y el síndrome de Sjögren. Puede estar presente en mieloma múltiple, tuberculosis, sífilis, lepra y cirrosis. Por eso su positividad no asegura el diagnóstico de artritis reumatoide y necesita correlacionarse con el cuadro clínico. Se determina por métodos de aglutinación usando partículas de poliestireno recubiertas con inmunoglobulina $G$ humana. (Castresana, 2004). Durante la Artritis Reumatoide los dos primeros años de evolución la incidencia del factor reumatoide es baja, de sólo un 30 a un $60 \%$ de los casos, y no es raro que desaparezca al remitir la enfermedad.

\section{Enfermedades asociadas al Factor Reumatoide}

La determinación del factor reumatoide por el método de aglutinación puede aparecer positivo en fermedades inmunitarias, entre ellas tenemos:

\section{Artritis Reumatoide (AR)}

Es una enfermedad sistémica autoinmune, crónica, cuya etiología es multifactorial y no existe una causa específica establecida. La expresión de la enfermedad podría obedecer a una combinación de aspectos inmunes, ambientales y genéticos. Puede presentarse a cualquier edad, pero la mayoría de los casos se encuentran entre la tercera y quinta décadas de la vida, afecta principalmente al sexo femenino. 
El diagnóstico es básicamente clínico y depende en gran parte de una historia adecuada y cuidadosa del paciente. Los tratamientos hacen énfasis en la necesidad de su implementación precoz, porque cuanto antes se trate, mayores son las posibilidades de buena respuesta, con el fin de prevenir el daño. (Restrepo, 2007).

El dolor en las articulaciones es el sistema más frecuente AR, este dolor es consecuencia de la inflamación, que con frecuencia se puede ver a simple vista. La AR afecta a cualquier articulación periférica y columna cervical, los pacientes con dolor en todas las articulaciones, la columna y músculos no son el prototipo de AR. Las articulaciones dañadas con más frecuencia son las muñecas, nudillos, articulaciones de los dedos, los codos, hombro, las caderas, rodillas, tobillos, y dedos de los pies.

Según el Ministerio de salud de Chile (2014), la inflamación y el dolor dan lugar a una rápida pérdida de tejido muscular en torno a la articulación afectada, lo que contribuye a la pérdida de la función articular. Si la inflamación de la membrana sinovial no se puede suprimir, el resultado será la aparición de daño articular erosivo, el que está relacionado con la intensidad y la duración de la inflamación; también, se debe considerar que otras estructuras tienen revestimientos sinoviales, tales como las vainas de los tendones, por tanto la inflamación de éstos pueden resultar en la rotura del tendón.

Desde las fases iniciales de la enfermedad afecta de manera significativa a las actividades diarias de las personas que la padecen, no solo en sus aspectos físicos, como son las actividades ocupacionales o de recreo, sino también en los aspectos sociales, psicológicos y económicos. Una vez que el diagnóstico se ha establecido y el paciente comprende el significado de la enfermedad, comienza un periodo de adaptación que también incluye a su entorno.

\section{Lupus Eritematoso Sistémico (LES)}

Es definido por sus características clínicas y por la presencia invariable de anticuerpos en la sangre dirigidos contra uno o más componentes de los núcleos celulares. Algunas manifestaciones parecen estar asociadas de dichos anticuerpos y con determinados marcadores genéticos. La LES es una sola enfermedad con una amplia gama de manifestaciones, cuya expresión puede estar influida por factores genéticos del paciente. Ocasionalmente los pacientes presentan rasgos de esta enfermedad y otras del tejido conectivo como artritis reumatoide y esclerodermia, cualquiera puede tener lupus, pero en la mayoría de los casos afecta a las mujeres.

La clave para poder vivir con lupus es estar informado acerca de la enfermedad, su impacto y tener un papel activo en el tratamiento; muchas personas tienen ciertos síntomas; tales como: dolor o inflamación de las articulaciones, músculos, fiebre inexplicable, sarpullido enrojecido más a menudo en la cara, pérdida de cabello, sensibilidad del sol, cansancio.

\section{Tuberculosis}

Es una infección producida por el Bacilo de Koch, se caracteriza por un periodo latente prolongado entre la infección inicial y las manifestaciones clínicas en la que predomina la neumopatía (aunque también puede afectar a otros órganos) y una respuesta granulo matosa con inflamación y lesión en los tejidos.

Se trasmite por inhalación de gotitas infecciosas, eliminadas al aire por estornudos de un paciente con tuberculosis. La transmisión puede ser indirecta; ya que, la micobacteria es muy resistente a la desecación y puede estar muchos meses en el polvo u objeto de uso diario. Las principales puertas de entrada son: el sistema respiratorio, el tejido linfoide de la bucofaringe el intestino y la piel; la vía de contagio más común es la respiratoria. Los síntomas pueden serfatiga, pérdida de 
peso, astenia, sudoraciones nocturnas, fiebre vespertina, tos, expectoración y la disnea. La infección inicial suele ser asintomática y a las pocas semanas desarrolla sensibilidad a la prueba. Sin tratamiento los enfermos mueren en un periodo aproximado de dos años, pero con tratamiento en un corto periodo de tiempo (2-3 semanas) el enfermo tiene una alta probabilidad de curación. (Morán \& Lazo, 2007).

\section{Síndrome de Sjögren (SS)}

Es una enfermedad autoinmune crónica que se caracteriza por la presencia de manifestaciones clínicas ocasionada por la afectación de las glándulas exocrinas y en ocasiones por afectación extraglandular que va a condicionar el pronóstico.

El SS ocurre en paciente de todas las edades, pero afecta sobre todo a mujeres entre 40-50 años. La principal clínica consiste en la sequedad de mucosas sobre todo ocular y oral, pero existe un amplio abanico de síntomas como: parotidomegalia, fiebre, purpura palpable, neumonitis intersticial, probablemente la enfermedad autoinmune más frecuente, pero poco diagnosticada.

\section{Mononucleosis Infecciosa}

Es una enfermedad producida por el virus de EpsteinBarr, clínicamente hay que destacar la existencia de un periodo de incubación que oscila entre 3 y 7 semanas, la duración de la fase sintomática oscila entre 2 y 4 semanas; en niños menores de cinco años la infección suele ser asintomática; aunque la enfermedad afecta a niños de más edad y adolescentes, es posible la aparición de síntomas. Entre las manifestación más comunes: fiebre, linfadenopatías y faringitis; la esplenomegalia, petequias en paladar y hepatomegalia se presenta en más del 10\% de pacientes. En los adultos y jóvenes la infección tiene lugar a través de la saliva, al besarse con alguien infectado; por el contrario, en los niños espacialmente en aquellos que acuden a guarderías, el contagio se produce debido al contacto que hay en sus actividades rutinarias; el hombre es el único reservorio natural para este virus. Restrepo,(2007)

\section{Infecciones Virales}

Las infecciones virales son difíciles de tratar, porque los virus viven dentro de las células del organismo. Están protegidos contra los medicamentos, que suelen trasladarse a través del torrente sanguíneo, en algunos cosos los antibióticos no surten efectos para combatir las infecciones, existen apenas unos cuantos medicamentos antivirales disponibles incluyendo las vacunas que pueden evitar muchas infecciones virales. (Jiménez, 2012).

\section{Diagnóstico}

Los métodos utilizados para reconocer estas enfermedades pueden clasificarse en directos e indirectos. Gran parte de las técnicas utilizadas en el diagnóstico clínico se basan en pruebas serológicas que identifican anticuerpos específicos frente a diversas proteínas antigénicas.

\section{Inmunofluorecencia Directa (ID)}

Es una de las técnicas más antiguas y de uso más difundido en el laboratorio clínico. El principio básico de esta técnica se puede utilizar para una identificación rápida directamente sobre la muestra. El advenimiento de los anticuerpos monoclonales, ha incrementado la especificidad y en algunos casos la sensibilidad de estos ensayos.

\section{Test de Aglutinación}

Es un método simple, que a veces se usa para la detección de antígenos virales en muestras clínicas. Los ensayos de aglutinación, dependen de la fijación inicial de anticuerpos antivirales específicos sobre eritrocitos o partículas de látex. Luego este reactivo se 
incuba con la muestra clínica en la cual se investiga el antígeno y las partículas se aglutinan si el antígeno adecuado se encuentra presente. Estas pruebas en general se complementan o se confirman por medio de otros ensayos debido al elevado porcentaje de reacciones inespecíficas.

\section{Enzimoinmunoanálisis (EIA)}

Los EIA para la detección de antígeno se basan habitualmente en la captura del antígeno por anticuerpos específicos unidos a una fase sólida, en general el pocillo de una microplaca o una pequeña esfera de plástico. El antígeno viral presente en la muestra clínica se combina con el anticuerpo fijado a la fase sólida y el antígeno viral se detecta mediante la adición de otro anticuerpo específico conjugado a una enzima. La enzima conjugada suele ser peroxidasa o fosfatasa alcalina. El substrato para esas enzimas varía. En la reacción con la peroxidasa el substrato es un peróxido capaz de oxidar un compuesto químico incoloro que en su forma oxidada tiene un color característico. (Sandin, 2009).

\section{MATERIALES Y MÉTODOS}

\section{Tipo de investigación}

Según (Bernal, 2006), es una investigación analítica; porque, consiste en descomponer un objetivo de estudio separando cada una de las partes del todo para estudiarla en forma individual. Cuantitativo porque usa la recolección de datos para probar hipótesis con base en la numeración y el análisis estadístico, para establecer patrones de comportamientos y probar teorías. (Sampieri \&Fernández \& Lucio, 2010).

\section{Tipo de estudio}

Según (Bernal, 2006), es descriptivo porque se reseñan las características o rasgos de la situación o fenómeno u objeto de estudio; transversal porque recolecta datos en un solo momento, en un tiempo único. (Sampieri et al, 2010).

\section{Área de estudio}

Según la alcaldía municipal, el barrio Nuevo Amanecer que se encuentra en la cuidad de Juigalpa Chontales, teniendo sus límites al norte con el barrio Héctor Ugarte, al sur con el rio la Pachona, al este la terminal de autobuses Mayales y estadio de béisbol Carlos Guerra Colindres, al oeste con el barrio Padre Miguel; habitan alrededor de 435 familias, con un promedio de habitantes de 2, 175 .

\section{Universo}

Está conformado por las mujeres que tengan las edades de 40-60 y que sean habitantes del barrio Nuevo Amanecer de la ciudad de Juigalpa, Chontales.

\section{Muestra}

Está representada por mujeres que comprenden las edades en estudio y que habiten en el barrio antes mencionado, al mismo tiempo que estén dispuestas a participar en la investigación.

\section{Tipo de muestreo}

Debido a que no tomaremos a todas las mujeres que habitan en el barrio y solo a las de 40-60 años que estén dispuestas a participar en el estudio es un muestreo no probabilístico por conveniencia.

\section{Técnica e instrumento de recolección de datos}

El instrumento que se empleó para la recolección de datos fue una encuesta con preguntas cerradas, en la cual abordamos 7 interrogantes relacionadas al tema de estudio. 


\section{Procesamiento de datos}

Para procesar los datos hicimos uso de los programas electrónicos Word y SPSS21 donde se procesó la encuesta para sacar con exactitud los porcentajes.

El análisis de las muestras se llevó a cabo en el laboratorio Clínico Docente de la Farem Chontales y la recolección de las muestras se realizó casa a casa en el barrio en estudio.

\section{RESULTADOS Y DISCUSIÓN}

Presentación de los Resultados de la encuesta aplicada y los análisis obtenidos de las muestras serológicas de las mujeres de 40-60 años del barrio Nuevo Amanecer de la ciudad de Juigalpa, Chontales.

\begin{tabular}{lrrr}
\hline \multicolumn{1}{c}{ Oficios } & \multicolumn{1}{c}{$\begin{array}{c}\mathbf{N}^{\circ} \text { de } \\
\text { participantes }\end{array}$} & Porcentaje & $\begin{array}{r}\text { Porcentaje } \\
\text { acumulado }\end{array}$ \\
\hline Ama de & 13 & 32.5 & 32.5 \\
Casa & 1 & 2.5 & 35.0 \\
Docente & 1 & 2.5 & 37.5 \\
Enfermera & 1 & 2.5 & 40.0 \\
Halar Leña & 3 & 7.5 & 47.5 \\
Secretaria & 5 & 12.5 & 60.0 \\
Tortillera & 16 & 40.0 & 100.0 \\
Asistente & & & \\
del hogar & $\mathbf{4 0}$ & $\mathbf{1 0 0 . 0}$ & \\
\hline Total & & & \\
\hline
\end{tabular}

El 100\% de los participantes en el estudio son mujeres procedentes del Barrio nuevo amanecer de Juigalpa Chontales encontrándose un mayor porcentaje con ocupación asistente del hogar que corresponde al $40 \%$ de los encuestados.

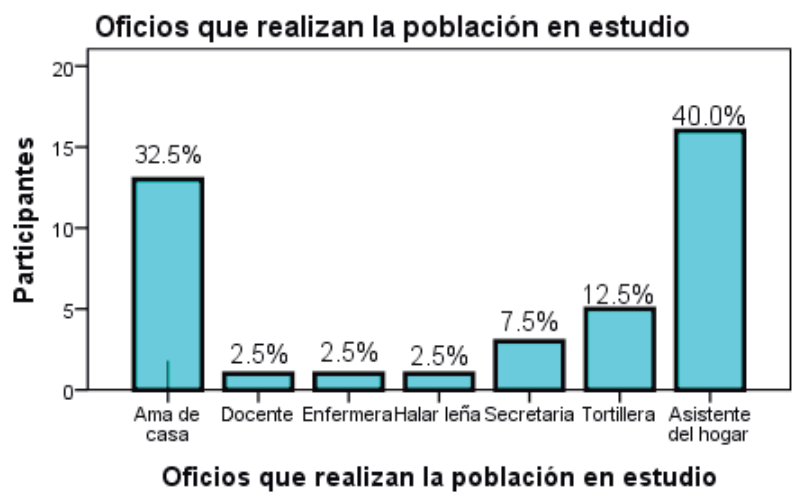

De acuerdo a los análisis de las muestras sanguíneas obtenidas; en donde, por centrifugación se obtuvo el espécimen (suero), que fue lo que se utilizó para la realización del examen de Factor Reumatoide, estas muestras fueron recolectadas de mujeres que comprendían las edades de 40 a 60 que habitan en el barrio Nuevo Amanecer de la ciudad de Juigalpa, Chontales, durante la realización del examen por el método Cualitativo según los intervalos de edades estudiadas que fueron de 40-45 , 46-50,51-60 años, el $100 \%$ de la muestras serológicas en estudio se encontraron negativas al momento de la realización del test.

El haber encontrado todas las muestras negativas, indica que la población en estudio no presenta anticuerpos de factor reumatoide en el periodo de tiempo que se realizó la investigación, no obstante esto no descarta la probabilidad que desarrollen artritis reumatoide en el futuro, debido a que se ha demostrado que la enfermedad temprana puede permanecer al principio indiferenciada y persistir así algún tiempo, es por ello lo que su identificación es importante por su gran impacto tanto para el individuo como para la sociedad ; y la población de este barrio presentan factores predisponentes a esta patología ya que, desempeñan labores como: Ama de casa (32.5\%), Docente(2.5\%), Enfermera (2.5\%), Halar leña (2.5\%), Secretaria $(7.5 \%)$, Tortillera $(12.5 \%)$, Asistente del hogar (40\%), los cuales son factores desencadenantes de la enfermedad, podría obedecer a una combinación de aspectos inmunes, ambientales y genéticos; en donde las articulaciones dañadas con más frecuencia son las muñecas, nudillos, articulaciones de los dedos, los codos, hombro, las caderas, rodillas, tobillos, y dedos de los pies . (Restrepo, 2007).

Según Neira\& Ortega, (2006) actualmente no hay consenso sobre el tiempo transcurrido desde el inicio de los síntomas para considerar el estado temprano de la enfermedad. 
Concluyendo de esta manera que en este estudio hubo un predominio del sexo femenino pues se conoce en la actualidad que la incidencia de esta enfermedad aumenta sorprendentemente durante la vida adulta hasta los 85 años, donde esta incidencia es doble en las mujeres con respecto a los hombres.

En este estudio no se tomó en cuenta la variable color de piel debido a que en nuestro país existe una gran mezcla racial, puesto que estimaciones más altas referente a la raza corresponden a las tribus indias americanas y esquimales por encima del 3\% y las más bajas se han encontrado en África y Asia por debajo del 0.2\%.Carmona (2002).

El método usado para el estudio fue el de aglutinación en látex para la determinación cualitativa $\mathrm{y}$ semicuantitativa del factor reumatoide en suero, HUMATEX RF, que esta estandarizado para detectar concentraciones de RF en muestras de suero no diluido para aproximadamente $12 \mathrm{Ul} / \mathrm{ml}$ lo que indica que las muestras serológicas presentaron concentraciones menores a este valor de referencia $\mathrm{Ul} / \mathrm{ml}$.

Nuestra investigación nos permite concluir que más de la mitad de los pacientes presentaron síntomas de dolores articulares sin embargo el resultado del test en el total de los estudiados resultó negativo orientándole a la población que aceptó participar en el estudio, que se estén realizando continuamente chequeos médicos incluyendo este tipo de examen debido a que esta enfermedad suele presentarse de forma gradual y lentamente progresiva. Padecer una enfermedad crónica como es la Artritis reumatoide (AR) tiene consecuencias importantes para las personas afectadas pero también para la sociedad, siendo este el principal objetivo del estudio prevenir a la población carente de información de esta enfermedad que una vez identificada puede ayudar a las personas a mejorar su calidad de vida detectándose en lo más rápido posible, los tratamientos hacen énfasis en la necesidad de su implementación precoz, porque cuanto antes se trate, mayores son las posibilidades de una respuesta satisfactoria, es por tal razón que consideramos este estudio con un gran aporte científico, social y humanitario para nuestro Pais.

\section{REFERENCIAS BIBLIOGRÁFICAS}

Artritis reumatoide. (Junio de 2014). Ministerio de salud. Obtenido de http://www.web.minsa.cl/sites/ defaul/files/GPC\%20Artritis.pdf

Bernal, C. A. (2006). Metdología de la Investigación. México: Cámara Nacional de la Industria editorial Méxica.

Castresana, C. (2004). La interpretación del Laboratorio Clínico en reumatología. Obtenido de http://www. binasss.sa.cr/revistas/rmcc/566/articulo6.pdf

Carmona L. Epidemiología de la artritis reumatoide. Rev Esp Reumatol. 2002;29(3):86-9.

Elena Morán López \& Yaima Lazo Amador. (Enero de 2007). Tuberculosis. Revista Cubana de Estomatología. Obtenido de http://www. site.ebrary.com/lib/bibliotecaunansp/detail. action?docID $=10174866 \& p 00=$ tuberculosis

Goldsby \& Kindt \& Osborne \& Kudy, (2004). Inmunologia Clinica

Gauehff, A. (2007). Respuesta Inmuno: Anticurpo, Alergia, Vacuna y reproducción Humana. Argentina Eudeba. Obtenido: dehttp://site. ebrary.com/lib/bibliotecaunansp/detail. action?docID $=10357365 \& \mathrm{P} 00=$ Imnunología

José Gómez Puerta \& Ricard Cervera. (Abril de 2008). Lupus eritomatoso sistémico. La clínica del laboratorio, 14. Obtenido de http://www. medigraphic

Jiménez, J. R. (2012). Infectología Clínica (segunda ed.). México: Manual Moderno. http://www. site.ebrary.com/lib/bibliotecaunansp/reader. action?docID $=10820480 . \mathrm{com} / \mathrm{pdfs} / \mathrm{medlab} /$ myl085-6b.pdf

Neira, F., \& Ortega, J. L. (2006). Tratamiento del dolor en la artritis reumatoide fundamentado en medicina basada en la evidencia. Revista de la Sociedad 
Española del Dolor, 13(8), 561-566.

Restrepo, J. F. (2007). La clínica y el laboratorio. Obtenido de http://www.ditorial.utalca.cl/docs/ ebook

Roberto Hernández Sampieri \& Carlos Fernández Collado \& Pilar Baptista Lucio. (2010). Metodología de la Investigación (5 ed.). (E. C. S.A, Ed.) México: Cámara Nacional de la Industria Mexicana.
Sandin,M.D.(2009).htttp://www.vet.unicen.edu. ar/actividadescurriculares/virologia/images/ documentos/2009/diagnostico\%20viral.pdf

Ulises Mendoza Coussette \& María Eugenia\& Alonso Biosca. (2015). Rheumatoid factor. Association with atherogenic risk markers in rheumatoid arthristis patients. Obtenido de http://www.redalyc. org/articulo.oa?id=451644520009

Vidal,Irma(2010). Temas de Laboratorio Clinico. 